\title{
LONG-RANGE DEPENDENCE AND APPELL RANK
}

\author{
By Donatas SURGAILIS
}

\section{Vilnius Institute of Mathematics and Informatics}

\begin{abstract}
We study limit distributions of sums $S_{N}^{(G)}=\sum_{t=1}^{N} G\left(X_{t}\right)$ of nonlinear functions $G(x)$ in stationary variables of the form $X_{t}=Y_{t}+Z_{t}$, where $\left\{Y_{t}\right\}$ is a linear (moving average) sequence with long-range dependence, and $\left\{Z_{t}\right\}$ is a (nonlinear) weakly dependent sequence. In particular, we consider the case when $\left\{Y_{t}\right\}$ is Gaussian and either (1) $\left\{Z_{t}\right\}$ is a weakly dependent multilinear form in Gaussian innovations, or (2) $\left\{Z_{t}\right\}$ is a finitely dependent functional in Gaussian innovations or (3) $\left\{Z_{t}\right\}$ is weakly dependent and independent of $\left\{Y_{t}\right\}$. We show in all three cases that the limit distribution of $S_{N}^{(G)}$ is determined by the Appell rank of $G(x)$, or the lowest $k \geq 0$ such that $a_{k}=\partial^{k} E\left\{G\left(X_{0}+c\right)\right\} /\left.\partial c^{k}\right|_{c=0} \neq 0$.
\end{abstract}

1. Introduction and the main results. A strictly stationary time series $X_{t}, t \in \mathbf{Z}$ is said to be long-range dependent (LRD) if its covariance function $r(t)=\operatorname{Cov}\left(X_{0}, X_{t}\right)$ is not summable and decreases as a power of the lag; more precisely, if

$$
r(t)=L(t) t^{-\theta}
$$

$t \geq 1$, where $\theta \in(0,1)$ and $L(x)$ is a function, slowly varying at infinity. In the last decade, there has been considerable interest in LRD processes and statistical inference for such processes; see, for example, Beran (1992) and the references therein. There, many problems deal with the existence and description of limit distributions of sums

$$
S_{N}^{(G)}(t)=\sum_{s=1}^{[N t]} G\left(X_{s}\right), \quad t \geq 0,
$$

where $G(x), x \in \mathbf{R}$ is a (nonlinear) function with $E\left\{G\left(X_{0}\right)\right\}=0, E\left\{G^{2}\left(X_{0}\right)\right\}<$ $\infty$. For Gaussian LRD process $X_{t}$, this problem was first considered by Rosenblatt (1961) and later solved in full generality by Dobrushin and Major (1979) and Taqqu (1979), who showed that the limit in distribution of suitably normalized sums $S_{N}^{(G)}(t)$ (1.2) is determined by the Hermite rank $k^{*}=1,2, \ldots$ of $G(x)$, or the index of the first nonzero coefficient in the Hermite expansion

$$
G(x)=\sum_{k=0}^{\infty} \frac{g_{k}}{k !} H_{k}(x ; \sigma)
$$

Received October 1997; revised July 1999.

AMS 1991 subject classifications. Primary 60F05; secondary 60G15, 62M10.

Key words and phrases. Long-range dependence, noncentral limit theorems, reduction principle, Appell polynomials, Appell rank. 
in Hermite polynomials $H_{k}(x ; \sigma)=(-1)^{k} \sigma^{2 k} \exp \left(x^{2} / 2 \sigma^{2}\right) d^{k}\left(\exp \left(-x^{2} / 2 \sigma^{2}\right)\right) /$ $d x^{k}, k=0,1, \ldots$, where $\sigma^{2}=E\left\{X_{0}^{2}\right\}$. Namely, if $\theta k^{*}<1$, then

$$
D_{N, k^{*}}^{-1} S_{N}^{(G)}(t) \Rightarrow g_{k^{*}} \mathscr{H}_{k^{*}}(t),
$$

where $D_{N, k}=c(k, \theta) L(N)^{k / 2} N^{1-\theta k / 2}, c(k, \theta)=\{2 /(k !(1-k \theta)(2-k \theta))\}^{1 / 2}, \Rightarrow$ stands for weak convergence of finite-dimensional distributions, and $\mathscr{H}_{k}(t)$ is the Hermite process of order $1 \leq k<1 / \theta$ (see Section 2.5 for the definition). It was proved somewhat later [Breuer and Major (1983), Giraitis and Surgailis (1985)] that in the case $\theta k^{*}>1$, and more generally, if $\sum_{t \in \mathbf{Z}} \mid \operatorname{Cov}\left(G\left(X_{0}\right)\right.$, $\left.G\left(X_{t}\right)\right) \mid<\infty$, then $S_{N}^{(G)}(t)$ converge to the Brownian motion, under the usual $\sqrt{N}$-normalization.

However, the "nonlinear LRD-behavior" of non-Gaussian processes is much less understood. One of the most studied models of non-Gaussian LRD processes is the linear (moving average) process,

$$
X_{t}=\sum_{i \geq 0} b_{i} \zeta_{t-i}, \quad t \in \mathbf{Z},
$$

where $\zeta_{i}, i \in \mathbf{Z}$ is an i.i.d. sequence with zero mean and variance 1 , and $b_{i}, i \geq 0$ are (deterministic) weights of the form

$$
b_{i}=L_{1}(i) i^{-(1+\theta) / 2}, \quad i \geq 1,
$$

where $\theta \in(0,1)$ and where $L_{1}(x)$ is a function slowly varying at infinity. Linear processes of the type (1.5)-(1.6) include several important parametric classes such as fractional ARIMA and differenced fractional noise.

Limit distributions of sums $S_{N}^{(G)}(t)$ of polynomials $G(x)$ of linear process (1.5)-(1.6) were first studied by Surgailis (1982). It turned out that these distributions are the same as in the Gaussian case, with the only difference that the Hermite rank $k^{*}$ of $G(x)$ has to be replaced by the lowest $k \geq 0$ such that

$$
a_{k} \equiv E\left\{G^{(k)}\left(X_{0}\right)\right\} \neq 0,
$$

where $G^{(k)}(x)=d^{k} G(x) / d x^{k}$. Later, Giraitis and Surgailis (1986, 1989), Avram and Taqqu (1987) observed that $a_{k}$ are related to the Appell expansion

$$
G(x)=\sum_{k \geq 0} \frac{a_{k}}{k !} A_{k}(x)
$$

in Appell polynomials $A_{k}(x), k=0,1, \ldots$, defined by the formal power series

$$
\sum_{k \geq 0} \frac{z^{k}}{k !} A_{k}(x)=\frac{e^{z x}}{E\left\{e^{z X_{0}}\right\}} .
$$

Recently, limit distribution of sums and quadratic forms of Appell polynomials in linear variables (1.5)-(1.6) was studied by Giraitis and Taqqu (1997), Giraitis, Taqqu and Terrin (1998). On the other hand, the asymptotics of $S_{N}^{(G)}(t)$ for nonsmooth $G(x)$, in particular for indicator functions $G_{y}(x)=$ $\mathbf{1}(x \leq y), y \in \mathbf{R}$ of linear process (1.5)-(1.6) were obtained by Ho and Hsing (1996), Koul and Surgailis (1997). The above-mentioned papers provide a 
number of important applications of the limit theorems to statistical inference. However, the applicability of these results is limited to linear or Gaussian LRD time series, which is often unrealistic or hard to verify in practice.

The natural question is what happens if the LRD process $X_{t}$ is nonlinear, in particular, if the Appell expansion (1.8) can be used to characterize scaling limits of $S_{N}^{(G)}(t)$ as above. Such a possibility is not obvious, since $A_{k}(x), k \geq$ 0 depend on the marginal distribution of $X_{t}$ and hence on its "short-range dependent" behavior, which seems unrelated to long-range dependence.

In this paper we study this question in the case when the underlying process $X_{t}$ can be written as

$$
X_{t}=Y_{t}+Z_{t}, \quad t \in \mathbf{Z},
$$

where $Y_{t}, t \in \mathbf{Z}$ is a linear LRD process of the form (1.5)-(1.6) and $Z_{t}, t \in \mathbf{Z}$ is a nonlinear weakly dependent process of a certain type. More concretely, we consider three types of the weakly dependent component $Z_{t}$ in (1.10), for which we can prove the limit distribution of $S_{N}^{(G)}(t)$ (Theorems 1-3 below). In all three cases, this distribution is of the same type as above and is determined by

$$
k^{*}=\min \left\{k \geq 0: a_{k} \neq 0\right\},
$$

where

$$
a_{k}=\partial^{k} E\left\{G\left(X_{0}+c\right)\right\} /\left.\partial c^{k}\right|_{c=0} .
$$

We call $k^{*}(1.11)$ the Appell rank of $G(x)$. It is clear that if $G(x)$ is a polynomial, then (1.12) coincides with (1.7). It is easy to show that if $X_{0} \sim \mathscr{N}\left(0, \sigma^{2}\right)$ then $a_{k}$ (1.12) coincide with $g_{k}$ in the Hermite expansion (1.3) and, in this case, Appell and Hermite ranks coincide.

Let us formulate the main results of the paper. We assume below that $Y_{t}, t \in \mathbf{Z}$ is a linear process

$$
Y_{t}=\sum_{i \geq 0} b_{i} \zeta_{t-i}, \quad t \in \mathbf{Z},
$$

where $b_{i}$ are given by (1.6) and $\zeta_{i}, i \in \mathbf{Z}$ are i.i.d. random variables, with zero mean, variance 1 and finite moments of arbitrary order. Note $\operatorname{Cov}\left(Y_{0}, Y_{t}\right)=L_{2}(t) t^{-\theta}(t \geq 1)$, where $L_{2}(t)$ is a slowly varying function such that $\lim _{t \rightarrow \infty} L_{2}(t) / L_{1}^{2}(t)=c \in(0, \infty)$. Below, $D_{N, k}=c(k, \theta) L_{2}(N)^{k / 2} N^{1-k \theta / 2}$.

THEOREM 1. Let $X_{t}=Y_{t}+Z_{t}$, where $Y_{t}, t \in \mathbf{Z}$ is a linear LRD process of (1.13), and $Z_{t}, t \in \mathbf{Z}$ is a multilinear form

$$
Z_{t}=\sum_{k=1}^{n} \sum_{i_{1}, \ldots, i_{k} \in \mathbf{Z}} b_{i_{1}, \ldots, i_{k}}^{(k)} \zeta_{t-i_{1}} \cdots \zeta_{t-i_{k}}, \quad t \in \mathbf{Z}
$$

in the i.i.d. variables $\zeta_{i}, i \in \mathbf{Z}$, with summable coefficients

$$
\sum_{i_{1}, \ldots, i_{k} \in \mathbf{Z}}\left|b_{i_{1}, \ldots, i_{k}}^{(k)}\right|<\infty, \quad k=1, \ldots, n .
$$


Let $G(x)$ be a polynomial with $E\left\{G\left(X_{0}\right)\right\}=0, E\left\{G^{2}\left(X_{0}\right)\right\}<\infty$, and let $\theta k^{*}<$ 1. Then

$$
D_{N, k^{*}}^{-1} S_{N}^{(G)}(t) \Rightarrow a_{k^{*}} \mathscr{H}_{k^{*}}(t)
$$

THEOREM 2. Let $X_{t}=Y_{t}+Z_{t}$, where $Y_{t}, t \in \mathbf{Z}$ is a Gaussian LRD process of $(1.13), \zeta_{i} \sim \mathscr{N}(0,1)$ and let

$$
Z_{t}=V\left(\zeta_{t}, \zeta_{t-1}, \ldots, \zeta_{t-m}\right), \quad t \in \mathbf{Z}
$$

where $m<\infty$ and where $V\left(z_{0}, \ldots, z_{m}\right)$ is an arbitrary measurable function on $\mathbf{R}^{m+1}$.

Let $G(x)$ be an arbitrary measurable function with $E\left\{G\left(X_{0}\right)\right\}=0$, $E\left\{G^{2}\left(X_{0}\right)\right\}<\infty$, whose Appell rank $k^{*}$ satisfies $\theta k^{*}<1$. Then the convergence (1.16) holds true.

THEOREM 3. Let $X_{t}=Y_{t}+Z_{t}$, where $Y_{t}, t \in \mathbf{Z}$ is a Gaussian LRD process of (1.13), and $Z_{t}, t \in \mathbf{Z}$ is a strictly stationary sequence, independent of $Y_{t}, t \in \mathbf{Z}$ and such that, for any measurable function $\lambda(x)$ with $E\left\{\lambda^{2}\left(Z_{0}\right)\right\}<\infty$,

$$
\sum_{t \in \mathbf{Z}}\left|\operatorname{Cov}\left(\lambda\left(Z_{0}\right), \lambda\left(Z_{t}\right)\right)\right|<\infty \text {. }
$$

Let $G(x)$ satisfy the same conditions as in Theorem 2. Then the convergence (1.16) holds true.

REMARK 1. Note $E\left\{Z_{0}^{2}\right\}<\infty$ and $E\left\{X_{0}^{2}\right\}<\infty$ in Theorem 1. Moreover, condition (1.15) guarantees that $\left\{Z_{t}\right\}$ is weakly dependent in the sense that $\sum_{t \in \mathbf{Z}}\left|\operatorname{Cov}\left(Z_{0}, Z_{t}\right)\right|<\infty$.

REMARK 2. Theorems 2 and 3 do not assume any moment restrictions on the marginal distribution of $Z_{0}$. In particular, Theorem 3 is valid for stationary sequences of the form $X_{t}=Y_{t}+Z_{t}$, where $\left\{Y_{t}\right\}$ is a Gaussian LRD process of (1.13), and $\left\{Z_{t}\right\}$ is an arbitrary i.i.d. sequence, independent of $\left\{Y_{t}\right\}$. Clearly, this allows $E\left\{X_{0}^{2}\right\}=\infty$ and even $E\left\{\left|X_{0}\right|^{p}\right\}=\infty$ for each $p>0$. In such a case, the definition (1.1) of long-range dependence does not apply. As an indication of long-range dependence of $\left\{X_{t}\right\}$, one may consider the regular growth of the variance

$$
\operatorname{Var}\left(S_{N}^{(G)}\right) \approx a_{1}^{2} L_{2}(N) N^{2-\theta}, \quad N \rightarrow \infty,
$$

where $G(x)$ belongs to a certain class of square integrable functions $\left(E\left\{G^{2}\left(X_{0}\right)\right\}<\infty\right)$ of Appell rank 1 ; the symbol $\approx$ means "asymptotically proportional to." Relation (1.19) follows easily from the proofs of Theorems 1-3; see Corollary 4.1 below.

On the other hand, if $E\left\{Z_{0}^{2}\right\}<\infty$, then the covariance $r(t)=\operatorname{Cov}\left(X_{0}, X_{t}\right)$ is well defined, and one may ask if $X_{t}$ is LRD in the sense of (1.1). While this can be easily seen to be true for $X_{t}$ of Theorem 2 , in the remaining two situations (Theorems 1 and 3 ) additional assumptions on $Z_{t}$ seem necessary to 
guarantee the validity of (1.1). However, one can easily verify $\operatorname{Var}\left(\sum_{t=1}^{N} X_{t}\right) \approx$ $L_{2}(N) N^{2-\theta}$, under the premises of either Theorems 1,2 or 3 .

Theorems 1-3 show that the reduction principle by Taqqu (1979) holds for a large class of non-Gaussian processes. They also suggest that some results in asymptotic inference of LRD time series may be "structurally stable" with respect to certain additive nonlinear weakly dependent "perturbations" of a Gaussian or linear model. In particular, Theorem 2 implies the following central limit theorem for the empirical distribution function $F_{N}(x)=N^{-1} \sum_{t=1}^{N} \mathbf{1}\left(X_{t} \leq x\right)$ :

$$
N D_{N, 1}^{-1}\left(F_{N}(x)-F(x)\right) \Rightarrow F^{(1)}(x) W
$$

where $F(x)=P\left\{X_{0} \leq x\right\}$ and $W \sim \mathscr{N}(0,1)$. In (1.20), the dependence of the right-hand side in $x \in \mathbf{R}$ has exactly the same degenerate form as if $X_{t}, t \in \mathbf{Z}$ were Gaussian.

Theorems 1-3 are proved in Sections 3 and 4. The proof of Theorem 1 uses the formalism of (multivariate) Appell polynomials and their diagrams, which is explained in Section 2. In the case of Gaussian $Y_{t}, t \in \mathbf{Z}$, this formalism becomes the well-known diagram calculus of Itô-Wiener integrals; see, for example, Dobrushin (1979) or Major (1981). However, we do not want to restrict the discussion to the Gaussian case, because this case seems rather special, and the linearity of the LRD-part in (1.10) seems more important than Gaussianity.

2. Multivariate Appell polynomials and diagrams. In this section we discuss the formalism of multivariate Appell polynomials and diagrams. Most of the facts below can be found in Surgailis (1983) and Giraitis and Surgailis (1986). [See also Avram and Taqqu (1987), Giraitis and Taqqu (1997).] For the reader's convenience, we present the proofs of Lemmas 2.1-2.3 in an Appendix.

2.1. Multivariate Appell polynomials. From a notational point of view, it is more convenient to consider polynomials in random variables rather than in real variables. Let $Y_{j}, j=1,2, \ldots, n$ be a finite system of random variables. We shall assume that all moments of $Y_{1}, \ldots, Y_{n}$ are finite but no other conditions on their joint distribution is assumed. The multilinear form

$$
: Y_{1} Y_{2} \cdots Y_{n}:=\left.(-i)^{n} \frac{\partial^{n}}{\partial z_{1} \cdots \partial z_{n}}\left(\frac{\exp \left\{i \sum_{j=1}^{n} z_{j} Y_{j}\right\}}{E \exp \left\{i \sum_{j=1}^{n} z_{j} Y_{j}\right\}}\right)\right|_{z_{1}=\cdots=z_{n}=0}
$$

is called the Appell product of random variables $Y_{1}, \ldots, Y_{n}$. In particular, $: Y_{1}:=Y_{1}-E Y_{1},: Y_{1} Y_{2}:=Y_{1} Y_{2}-Y_{1} E Y_{2}-Y_{2} E Y_{1}+2 E Y_{1} E Y_{2}-E\left\{Y_{1} Y_{2}\right\}$. Let us note some easy properties of the Appell product. From the definition (2.1) it follows that the expectation of the Appell product is zero,

$$
E\left\{: Y_{1} \cdots Y_{n}:\right\}=0 .
$$

Furthermore, $: Y_{1} \ldots Y_{n}$ : is symmetric under permutations of $Y_{1}, \ldots, Y_{n}$. If $\left(Y_{1}, \ldots, Y_{k}\right)$ and $\left(Y_{k+1}, \ldots, Y_{n}\right)$ are independent, then

$$
: Y_{1} \cdots Y_{n}:=: Y_{1} \cdots Y_{k}:: Y_{k+1} \cdots Y_{n}:
$$


If $Y_{1}=\cdots=Y_{n}$, then $: Y \cdots Y: \equiv: Y^{, n}:=A_{n}(Y)$ is the Appell polynomial relative to the distribution of $Y$,

$$
A_{n}(Y)=\left.(-i)^{n} \frac{\partial^{n}}{\partial z^{n}}\left(\frac{e^{i z Y}}{E e^{i z Y}}\right)\right|_{z=0} .
$$

The multilinearity property of the Appell product means that, if $Y_{i}=$ $\sum_{j=1}^{m} a_{i j} Z_{j}, i=1 \ldots, n$ are linear combinations of random variables $Z_{1}, \ldots$, $Z_{m}$, then

$$
: Y_{1} \cdots Y_{n}:=\sum_{j_{1}=1}^{m} \cdots \sum_{j_{n}=1}^{m} a_{1, j_{1}} \cdots a_{n, j_{n}}: Z_{j_{1}} \cdots Z_{j_{n}}:
$$

We use the compact notation $: \prod_{i \in W} Y_{i}:=: Y^{,}$: for the Appell product of random variables $Y_{i}, i \in W$ indexed by the elements of a finite set $W$. Write $Y^{W}=\prod_{i \in W} Y_{i}$ for the usual product and $E\left\{Y^{W}\right\}=E\left\{\prod_{i \in W} Y_{i}\right\}$ and $\chi\left(Y^{, W}\right)=\chi\left(Y_{i}, i \in W\right)$ for the expectation and the joint cumulant, respectively, of random variables $Y_{i}, i \in W$. We shall distinguish between the notation $U \subset W$ and $U \subseteq W$ in the sense that the former will denote proper inclusion, that is, such that $U \neq W$. Put also $: Y^{\varnothing}:=Y^{\varnothing}=1$. Write $|W|$ for the number of elements of $W$, and $\mathscr{V}_{W}$ for the set of all partitions $\left(V_{1}, \ldots, V_{r}\right)$ of $W$ by nonempty subsets $V_{1}, \ldots, V_{r}, r=1,2, \ldots,|W|$.

LEMMA 2.1.

$$
\begin{aligned}
Y^{W} & =\sum_{U \subseteq W}: Y^{, U}: \sum_{\mathscr{Y} W \backslash U} \chi\left(Y^{, V_{1}}\right) \cdots \chi\left(Y^{, V_{r}}\right) \\
& =\sum_{U \subseteq W}: Y^{, U}: E\left\{Y^{W \backslash U}\right\},
\end{aligned}
$$

where the sum $\sum_{U \subseteq W}$ is taken over all subsets $U \subseteq W$ including $U=\varnothing$ and $U=W$.

2.2. Diagrams. Let us present a (diagram) formula which enables us to write the product $\prod_{i=1}^{k}: Y^{, W_{i}}$ : of Appell products as a sum of Appell products. Let $W_{1}, \ldots, W_{k}$ be mutually disjoint finite sets and $W=\bigcup_{i=1}^{k} W_{i}$. It is convenient to imagine $W$ as a table whose rows are $W_{1}, \ldots, W_{k}$. A diagram is a pair $\gamma=\left(U,(V)_{r}\right)$, where $U \subseteq W$ is a subset, and $(V)_{r} \equiv\left(V_{1}, \ldots, V_{r}\right) \in \mathscr{V}_{W \backslash U}$ is a partition of $W \backslash U$. The set $U$ will be called the free edge of $\gamma$ and the sets $V_{1}, \ldots, V_{r}$ will be called the connected edges of $\gamma$. A connected edge $V_{i} \subseteq W$ is said to be flat if it belongs to some (row) $W_{i}, i=1, \ldots, k$; in particular, any $V_{i} \subseteq W$ with $\left|V_{i}\right|=1$ is flat. A diagram $\gamma=\left(U,(V)_{r}\right)$ is said to be complete if $U=\varnothing$. Let $\Gamma_{W}$ and $\Gamma_{W}^{\dagger}$ denote the classes of all diagrams without flat edges and all complete diagrams without flat edges, respectively.

Let, as above, $Y_{j}, j \in W=\bigcup_{i=1}^{k} W_{i}$ be arbitrary collection of random variables with finite moments of any order. 
LEMMA 2.2.

$$
\begin{aligned}
& \prod_{i=1}^{k}: Y^{, W_{i}}:=\sum_{\gamma=\left(U,(V)_{r}\right) \in \Gamma_{W}}: Y^{, U}: \chi\left(Y^{, V_{1}}\right) \cdots \chi\left(Y^{, V_{r}}\right) \\
& =\sum_{U \subseteq W}: Y^{, U}: E\left\{\prod_{i=1}^{k}: Y^{,} W_{i} \backslash U:\right\} .
\end{aligned}
$$

Furthermore,

$$
E\left\{\prod_{i=1}^{k}: Y, W_{i}:\right\}=\sum_{\gamma \in \Gamma_{W}^{\dagger}} \chi\left(Y, V_{1}\right) \ldots \chi\left(Y, V_{r}\right) .
$$

2.3. Appell forms. Let $\zeta_{t}, t \in \mathbf{Z}$ be a sequence of i.i.d. random variables with zero mean, variance 1 and finite moments of arbitrary order. For any collection $\left(s_{1}, \ldots, s_{n}\right) \in \mathbf{Z}^{n}$, the Appell product

$$
: \zeta_{s_{1}} \cdots \zeta_{s_{n}}:=:\left(\zeta_{s_{1}^{\prime}}\right)^{k_{1}}: \cdots:\left(\zeta_{s_{k_{r}}^{\prime}}\right), k_{r}:
$$

provided $\left|\left\{s_{i}: s_{i}=s_{j}^{\prime}\right\}\right|=k_{j}, \quad j=1, \ldots, r, s_{i}^{\prime} \neq s_{j}^{\prime}(i \neq j), \quad \sum_{j=1}^{r} k_{j}=n$ hold; see (2.4). The product (2.9) has the following orthogonality property: for any collections $\left(s_{1}, \ldots, s_{n}\right) \in \mathbf{Z}^{n},\left(t_{1}, \ldots, t_{n^{\prime}}\right) \in \mathbf{Z}^{n^{\prime}}$ such that $\left\{s_{1}, \ldots, s_{n}\right\} \neq$ $\left\{t_{1}, \ldots, t_{n^{\prime}}\right\}$ as subsets of $\mathbf{Z}$,

$$
E\left\{: \zeta_{s_{1}} \cdots \zeta_{s_{n}}:: \zeta_{t_{1}} \cdots \zeta_{t_{n^{\prime}}}:\right\}=0
$$

Let $L^{p}\left(\mathbf{Z}^{n}\right)$ be the space of all real sequences $q=q\left(s_{1}, \ldots, s_{n}\right),\left(s_{1}, \ldots, s_{n}\right) \in$ $\mathbf{Z}^{n}$ such that $\|q\|_{L^{p}\left(\mathbf{Z}^{n}\right)}=\left(\sum_{s_{1}, \ldots, s_{n} \in \mathbf{Z}}\left|q\left(s_{1}, \ldots, s_{n}\right)\right|^{p}\right)^{1 / p}<\infty, 1 \leq p \leq \infty$. For any $q \in L^{2}\left(\mathbf{Z}^{n}\right)$ consider the polynomial form

$$
\mathscr{A}_{n}(q)=\sum_{s_{1}, \ldots, s_{n} \in \mathbf{Z}} q\left(s_{1}, \ldots, s_{n}\right): \zeta_{s_{1}} \cdots \zeta_{s_{n}}:
$$

By (2.10), the last sum converges in $L^{2}(\Omega)$; furthermore, for any $q, q^{\prime} \in L^{2}\left(\mathbf{Z}^{n}\right)$,

$$
\left|E\left\{\mathscr{A}_{n}(q) \mathscr{A}_{n}\left(q^{\prime}\right)\right\}\right| \leq C \sum_{s_{1}, \ldots, s_{n} \in \mathbf{Z}}\left|\operatorname{sym} q\left(s_{1}, \ldots, s_{n}\right) q^{\prime}\left(s_{1}, \ldots, s_{n}\right)\right|,
$$

where $\operatorname{sym} q\left(s_{1}, \ldots, s_{n}\right)=(n !)^{-1} \sum_{(p)_{n} \in \mathscr{P}_{n}} q\left(s_{p(1)}, \ldots, s_{p(n)}\right)$ is the symmetrization. We call $\mathscr{A}_{n}(q)$ (2.11) Appell form of order $n$. In particular,

$$
\begin{aligned}
& \mathscr{L}_{1}(q)=\sum_{s \in \mathbf{Z}} q(s) \zeta_{s}, \\
& \mathscr{L}_{2}(q)=\sum_{s_{1}, s_{2} \in \mathbf{Z}: s_{1} \neq s_{2}} q\left(s_{1}, s_{2}\right) \zeta_{s_{1}} \zeta_{s_{2}}+\sum_{s \in \mathbf{Z}} q(s, s)\left(\zeta_{s}^{2}-E \zeta_{s}^{2}\right) .
\end{aligned}
$$

By $(2.2), E\left\{\mathscr{A}_{n}(q)\right\}=0, n \geq 1$. Let $\mathscr{A}_{0}(q)=q \in \mathbf{R}$ be a scalar. As linear combinations of Appell polynomials : $\zeta_{s}^{, n}:, n=0,1, \ldots$ are dense in the space of all square integrable random variables measurable with respect to $\zeta_{s}$, by standard argument one can show that linear combinations of Appell forms 
$\mathscr{A}_{n}(q), q \in L^{2}\left(\mathbf{Z}^{n}\right), n \geq 0$ are dense in the space $L^{2}(\Omega)$ of all square integrable variables measurable with respect to the $\sigma$-algebra $\sigma\left\{\zeta_{s}: s \in \mathbf{Z}\right\}$.

Let us present a diagram formula for Appell forms similar to the diagram formula of Lemma 2.2.

Let $q_{i} \in L^{2}\left(\mathbf{Z}^{n_{i}}\right), n_{i} \geq 1, i=1, \ldots, k$ be given. Consider a function $\tilde{q} \in$ $L^{2}\left(\mathbf{Z}^{n}\right)$ of $n=n_{1}+\cdots+n_{k}$ variables $s_{i, j} \in \mathbf{Z}, i=1, \ldots, k, j=1, \ldots, n_{i}$, defined by

$$
\tilde{q}\left(s_{1,1}, \ldots, s_{k, n_{k}}\right)=q_{1}\left(s_{1,1}, \ldots, s_{1, n_{1}}\right) \cdots q_{k}\left(s_{k, 1}, \ldots, s_{k, n_{k}}\right) .
$$

It is convenient to write down the indices of the variables in (2.13) in the form of the table:

$$
W=\left(\begin{array}{c}
(1,1), \ldots,\left(1, n_{1}\right) \\
(2,1), \ldots,\left(2, n_{2}\right) \\
\ldots \\
(k, 1), \ldots,\left(k, n_{k}\right),
\end{array}\right)
$$

whose rows are denoted by $W_{i}, i=1, \ldots, k$. It is clear that there is a $1-$ 1 correspondence between table $W(2.14)$ and collection $(n)_{k}=\left(n_{1}, \ldots, n_{k}\right)$. Write $\Gamma_{W}=\Gamma_{(n)_{k}}, \Gamma_{W}^{\dagger}=\Gamma_{(n)_{k}}^{\dagger}$. Given a diagram $\gamma=\left(U,(V)_{r}\right) \in \Gamma_{(n)_{k}}$, the variables $s_{i, j}:(i, j) \in U$ will be said to be free, while $s_{i, j}:(i, j) \in V_{l}$ will be said to be connected, $l=1, \ldots, r$.

With each diagram $\gamma=\left(U,\left(V_{1}, \ldots, V_{r}\right)\right) \in \Gamma_{(n)_{k}}$ [and a given collection $\left.q_{i} \in L^{2}\left(\mathbf{Z}^{n_{i}}\right), i=1, \ldots, k\right]$ we associate a new function $q^{\gamma} \in L^{2}\left(\mathbf{Z}^{n_{\gamma}}\right)$, where $n_{\gamma}=|U|$ is the number of free variables, as follows. Replace in $\tilde{q}$ (2.13) all (connected) variables $s_{i, j}:(i, j) \in V_{l}$ by a single new variable $\tilde{s}_{l}, l=1, \ldots, r$ and denote the resulting function by $\tilde{q}^{\gamma}=\tilde{q}^{\gamma}\left(s_{i, j}, \tilde{s}_{l}:(i, j) \in U, l=1, \ldots, r\right)$. Then

$$
q^{\gamma}=q^{\gamma}\left(s_{i, j}:(i, j) \in U\right)=\prod_{l=1}^{r} \chi_{\left|V_{l}\right|} \sum_{\tilde{s}_{1}, \ldots, \tilde{s}_{r} \in \mathbf{Z}} \tilde{q}^{\gamma}\left(s_{i, j}, \tilde{s}_{l}\right),
$$

where $\chi_{k}=\chi_{k}\left(\zeta_{0}\right)$ is the $k$ th cumulant of $\zeta_{0}$. If $n_{\gamma}=0$ then $\gamma$ is complete and $q^{\gamma} \in \mathbf{R}$ is a scalar.

LEMMA 2.3.

$$
\begin{aligned}
\prod_{i=1}^{k} \mathscr{A}_{n_{i}}\left(q_{i}\right) & =\sum_{\gamma \in \Gamma_{(n)_{k}}} \mathscr{A}_{n_{\gamma}}\left(q^{\gamma}\right), \\
E\left\{\prod_{i=1}^{k} \mathscr{A}_{n_{i}}\left(q_{i}\right)\right\} & =\sum_{\gamma \in \Gamma_{(n)_{k}}^{\dagger}} q^{\gamma} .
\end{aligned}
$$

Consider now a particular case $n_{1}=\cdots=n_{m}=1,1 \leq m \leq k, q_{1}=$ $\cdots=q_{m} \equiv q \in L^{2}(\mathbf{Z}), \mathscr{A}_{1}(q) \equiv Y$. Let $A_{k}(x), k \geq 0$ be Appell polynomials corresponding to r.v. $Y$. From Lemma 2.3 the corollary follows. 
COROLlaRY 2.1.

$$
\begin{aligned}
Y^{m} & \prod_{i=m+1}^{k} \mathscr{A}_{n_{i}}\left(q_{i}\right) \\
& =\sum_{j=0}^{m}\left(\begin{array}{c}
m \\
j
\end{array}\right) A_{j}(Y) E\left\{Y^{m-j} \prod_{i=m+1}^{k} \mathscr{A}_{n_{i}}\left(q_{i}\right)\right\}+\sum_{\gamma \in \Gamma_{(n)_{k}^{m}}} \mathscr{A}_{n_{\gamma}}\left(q^{\gamma}\right),
\end{aligned}
$$

where $\Gamma_{(n)_{k}^{m}}$ consists of all diagrams $\gamma=\left(U,(V)_{r}\right) \in \Gamma_{(n)_{k}}$ such that $\bigcup_{i=m+1}^{k}$ $W_{i} \cap U \neq \varnothing$. [In other words, such that there is at least one free variable among the variables belonging to the functions $q_{m+1}, \ldots, q_{k}$ in (2.13).]

2.4. Gaussian case. Let $\zeta_{t}, t \in \mathbf{Z}$ be i.i.d. Gaussian $\mathscr{N}(0,1)$-distributed random variables. Then $\zeta_{t}=(2 \pi)^{-1 / 2} \int_{\Pi} e^{i t u} W(d u)$, where $\Pi=(-\pi, \pi]$, $W(d u)=\overline{W(-d u)}$ is a complex-valued random spectral measure on the real line with zero mean and variance $E\left\{|W(d u)|^{2}\right\}=d u$ ("Gaussian white noise"). The Appell product (2.9) can be written as

$$
: \zeta_{s_{1}} \cdots \zeta_{s_{n}}:=H_{k_{1}}\left(\zeta_{s_{1}^{\prime}}\right) \cdots H_{k_{r}}\left(\zeta_{s_{k_{r}}^{\prime}}\right)
$$

$H_{k}(x), k \geq 0$ being the standard Hermite polynomials, and the Appell form (2.11) as the $n$-tuple Itô-Wiener integral,

$$
\mathscr{A}_{n}(q)=\int_{\Pi^{n}} \hat{q}\left(u_{1}, \ldots, u_{n}\right) W\left(d u_{1}\right) \cdots W\left(d u_{n}\right),
$$

where $\hat{q}\left(u_{1}, \ldots, u_{n}\right)=(2 \pi)^{-n / 2} \sum_{s_{1}, \ldots, s_{n}} \exp \left\{i \sum_{j} u_{j} s_{j}\right\} q\left(s_{1}, \ldots, s_{n}\right)$ is the Fourier transform. The following orthogonality property $E\left\{\mathscr{A}_{n}(q) \mathscr{\mathscr { A }}_{n^{\prime}}\left(q^{\prime}\right)\right\}=$ $\delta_{n-n^{\prime}} n !\|q\|_{L^{2}\left(\mathbf{Z}^{n}\right)}^{2}, n, n^{\prime} \geq 0$ of multiple Itô-Wiener integrals is well known. The diagram formula of Lemma 2.3 in the "frequency representation" can be found, for example, in Dobrushin (1979), Proposition 4.1, with the important simplification that all edges $V_{l}$ connect only pairs, that is, $\left|V_{l}\right|=2, l=1, \ldots, r$.

2.5. Hermite processes. Let $\theta \in(0,1)$ be a parameter. We define a Hermite process of order $1 \leq k<1 / \theta$ as the $k$-tuple Itô-Wiener integral,

$$
\begin{aligned}
\mathscr{H}_{k}(t)=d_{k}(\theta) \int_{\mathbf{R}^{k}} & \frac{\exp \left(i t\left(u_{1}+\cdots+u_{n}\right)\right)-1}{i\left(u_{1}+\cdots+u_{n}\right)} \\
& \times \prod_{i=1}^{k}\left|u_{i}\right|^{(\theta-1) / 2} W\left(d u_{1}\right) \cdots W\left(d u_{k}\right), \quad t \geq 0,
\end{aligned}
$$

where the normalization factor $d_{k}(\theta)$ is chosen so that $E\left\{\mathscr{H}_{k}^{2}(1)\right\}=1$. See Taqqu $(1978,1979)$ for properties of Hermite processes.

3. Proof of Theorem 1. Observe first that $Z_{t}(1.14)$ can be rewritten as a sum of Appell forms,

$$
Z_{t}=\sum_{k=1}^{n} \sum_{s_{1}, \ldots, s_{k} \in \mathbf{Z}} q_{t-s_{1}, \ldots, t-s_{k}}^{(k)}: \zeta_{s_{1}} \cdots \zeta_{s_{k}}: \equiv \sum_{k=1}^{n} Z_{t}^{(k)}
$$


with some (new) coefficients $q^{(k)} \in L^{1}\left(\mathbf{Z}^{k}\right)$, that is, such that

$$
\sum_{s_{1}, \ldots, s_{k} \in \mathbf{Z}}\left|q_{s_{1}, \ldots, s_{k}}^{(k)}\right|<\infty, \quad k=1, \ldots, n .
$$

From (2.12) and (3.2) it follows that $Z_{t}^{(k)}, k=1, \ldots, n$ are weakly dependent in the sense that

$$
\sum_{t \in \mathbf{Z}}\left|E\left\{Z_{0}^{(k)}, Z_{t}^{(l)}\right\}\right|<\infty, \quad k, l=1, \ldots, n .
$$

Let $A_{k}(x), k \geq 0$ and $B_{k}(x), k \geq 0$ be Appell polynomials relative to the distributions of $X_{0}$ and $Y_{0}$, respectively. Theorem 1 follows from Surgailis (1982) and the following lemma.

Lemma 3.1. Let $G(x)$ be a polynomial of order $\ell \geq 1$. Then

$$
\sum_{t=1}^{N} G\left(X_{t}\right)=\sum_{k=k^{*}}^{\ell} \frac{a_{k}}{k !} \sum_{t=1}^{N} B_{k}\left(Y_{t}\right)+O_{P}(\sqrt{N}) .
$$

Proof. Consider first the case $G(x)=x^{k}$. By Corollary 2.1,

$$
\begin{aligned}
X_{t}^{k} & =\sum_{m=0}^{k}\left(\begin{array}{c}
k \\
m
\end{array}\right) Y_{t}^{m} Z_{t}^{k-m} \\
& =\sum_{m=0}^{k}\left(\begin{array}{c}
k \\
m
\end{array}\right) \sum_{j=0}^{m}\left(\begin{array}{c}
m \\
j
\end{array}\right) B_{j}\left(Y_{t}\right) E\left\{Y_{t}^{m-j} Z_{t}^{k-m}\right\}+R_{k, t},
\end{aligned}
$$

where the "remainder term" $R_{k, t}$ is given in (3.5) below. In order to identify this term, write

$$
Y_{0}^{m} Z_{0}^{k-m}=\sum_{(n)_{k}^{m}} \mathscr{L}_{1}^{m}(b) \mathscr{A}_{n_{m+1}}\left(q^{\left(n_{m+1}\right)}\right) \cdots \mathscr{A}_{n_{k}}\left(q^{\left(n_{k}\right)}\right)
$$

where $b=b_{i}, i \geq 0$ are given by (1.6) and the sum is taken over all collections $(n)_{k}^{m}=\left(n_{1}, \ldots, n_{m}, n_{m+1}, \ldots, n_{k}\right), 1 \leq n_{i} \leq n, 1 \leq i \leq k$ such that $n_{1}=\cdots=$ $n_{m}=1$. For each such collection $(n)_{k}^{m}$, put $q_{i}=b$ if $1 \leq i \leq m,=q^{\left(n_{i}\right)}$ if $m+1 \leq i \leq k$. Then $Y_{0}^{m} Z_{0}^{k-m}=\sum_{(n)_{k}^{m}} \prod_{i=1}^{k} \mathscr{A}_{n_{i}}\left(q_{i}\right)$. By applying Corollary 2.1 to the last product and taking the sum over all collections $(n)_{n}^{m}$, one obtains (3.4), with

$$
R_{k, t}=\sum_{m=0}^{k}\left(\begin{array}{c}
k \\
m
\end{array}\right) \sum_{(n)_{k}^{m}} \sum_{\gamma \in \Gamma_{(n)_{k}^{m}}^{m}} \mathscr{A}_{n_{\gamma}}\left(q_{t}^{\gamma}\right) .
$$

In (3.5), the last sum is the same as in (2.18) and $q_{t}^{\gamma}$ are defined as in (2.15), with the difference that $\tilde{q}$ of (2.13) must be replaced by the shifted function

$$
\tilde{q}_{t}\left(s_{1,1}, \ldots, s_{k, n_{k}}\right)=\tilde{q}\left(t+s_{1,1}, \ldots, t+s_{k, n_{k}}\right) .
$$


By changing the summation, (3.4) becomes

$$
\begin{aligned}
X_{t}^{k} & =\sum_{j=0}^{k} \frac{1}{j !} B_{j}\left(Y_{t}\right) \sum_{m=0}^{k-j} \frac{k !}{m !(k-j-m) !} E\left\{Y_{t}^{m} Z_{t}^{k-j-m}\right\}+R_{k, t} \\
& =\sum_{j=0}^{k} \frac{1}{j !} B_{j}\left(Y_{t}\right) \frac{k !}{(k-j) !} E\left\{X_{0}^{k-j}\right\}+R_{k, t} .
\end{aligned}
$$

Consequently, for any polynomial $G(x)=\sum_{k=0}^{\ell} d_{k} x^{k}$,

$$
G\left(X_{t}\right)=\sum_{j=0}^{\ell} \frac{1}{j !} E\left\{G^{(j)}\left(X_{t}\right)\right\} B_{j}\left(Y_{t}\right)+R_{t}=\sum_{j=k^{*}}^{\ell} \frac{a_{j}}{j !} B_{j}\left(Y_{t}\right)+R_{t},
$$

where

$$
R_{t}=\sum_{k=0}^{\ell} d_{k} R_{k, t}=\sum_{k=0}^{\ell} d_{k} \sum_{m=0}^{k}\left(\begin{array}{c}
k \\
m
\end{array}\right) \sum_{(n)_{k}^{m}} \sum_{\gamma \in \Gamma_{(n)_{k}^{m}}} \mathscr{A}_{n_{\gamma}}\left(q_{t}^{\gamma}\right) .
$$

Now, Lemma 3.1 follows from (3.7) and Lemma 3.2.

LEMMA 3.2. Under the conditions of Theorem 1,

$$
\operatorname{Var}\left(\sum_{t=1}^{N} R_{t}\right)=O(N)
$$

PROOF. As all sums on the right-hand side of (3.8) are finite, it suffices to show the lemma with $R_{t}$ replaced by $\mathscr{A}_{n_{\gamma}}\left(q_{t}^{\gamma}\right)$, for any diagram $\gamma$ as in (3.8). This follows from

$$
\sum_{t \in \mathbf{Z}}\left|E\left\{\mathscr{A}_{n_{\gamma}}\left(q_{0}^{\gamma}\right), \mathscr{A}_{n_{\gamma}}\left(q_{t}^{\gamma}\right)\right\}\right|<\infty
$$

or

$$
\sum_{t \in \mathbf{Z}} \sum_{s_{1}, \ldots, s_{n_{\gamma}} \in \mathbf{Z}}\left|\operatorname{sym} q_{0}^{\gamma}\left(s_{1}, \ldots, s_{n_{\gamma}}\right) q_{t}^{\gamma}\left(s_{1}, \ldots, s_{n_{\gamma}}\right)\right|<\infty
$$

see (2.12). We claim that, for each diagram $\gamma \in \Gamma_{(n)_{k}^{m}}$ as in (3.8), the function $q^{\gamma} \equiv q_{0}^{\gamma}$ has the following representation

$$
\begin{aligned}
& q^{\gamma}\left(s_{i, j}:(i, j) \in U\right) \\
& \quad=h^{\gamma}\left(s_{i, j}:(i, j) \in \bigcup_{l=m+1}^{k} W_{l} \cap U\right) \prod_{(i, j) \in \bigcup_{l=1}^{m} W_{l} \cap U} b\left(s_{i, j}\right),
\end{aligned}
$$

where $b(t) \equiv b_{t}$ are given in (1.6), $h^{\gamma} \in L^{1}\left(\mathbf{Z}^{\tilde{n}_{\gamma}}\right)$, and where $\tilde{n}_{\gamma}=\mid\{(i, j) \in$ $\left.\bigcup_{l=m+1}^{k} W_{l} \cap U\right\} \mid$ is the number of free variables among the rows $W_{m+1}, \ldots, W_{k}$ of the table $W$. 
Let us check that (3.10) implies (3.9). Indeed, with $n \equiv n_{\gamma}, \tilde{n} \equiv \tilde{n}_{\gamma}, \tilde{m}=$ $n-\tilde{n}, h \equiv h^{\gamma}$, for any permutation $(p)_{n} \equiv\left(p_{1}, \ldots, p_{n}\right) \in \mathscr{P}_{n}$, one obtains

$$
\begin{aligned}
I_{(p)_{n}} \equiv \sum_{t} \sum_{s_{1}, \ldots, s_{n}} \mid & b\left(s_{p_{1}}\right) \cdots b\left(s_{p_{\tilde{m}}}\right) h\left(s_{p_{\tilde{m}+1}}, \ldots, s_{p_{n}}\right) \\
& \times b\left(t+s_{1}\right) \cdots b\left(t+s_{\tilde{m}}\right) h\left(t+s_{\tilde{m}+1}, \ldots, t+s_{n}\right) \mid \\
=\sum_{t} \sum_{s_{1}, \ldots, s_{n}} \mid & b\left(s_{1}\right) \cdots b\left(s_{r}\right) h^{\prime}\left(s_{r+1}, \ldots, s_{r+\tilde{n}}\right) b\left(s_{r+\tilde{n}+1}\right) \cdots b\left(s_{n}\right) \\
& \times b\left(t+s_{1}\right) \cdots b\left(t+s_{\tilde{m}}\right) h\left(t+s_{\tilde{m}+1}, \ldots, t+s_{n}\right) \mid,
\end{aligned}
$$

for some $0 \leq r \leq \tilde{m}$ and $h^{\prime} \in L^{1}\left(\mathbf{Z}^{\tilde{n}}\right)$, which depend on $(p)_{n}$. Clearly, it suffices to consider $\bar{r}=\overline{0}$ only. If $\tilde{\tilde{m}}<\tilde{n}$, then $I_{(p)_{n}} \leq\|b\|_{L^{\infty}(\mathbf{Z})}^{2 \tilde{m}}\|h\|_{L^{1}\left(\mathbf{Z}^{\tilde{n}}\right)}\left\|h^{\prime}\right\|_{L^{1}\left(\mathbf{Z}^{\tilde{n}}\right)}<\infty$. If $\tilde{n}<\tilde{m}$, then $I_{(p)_{n}} \leq\|b\|_{L^{2}(\mathbf{Z})}^{2(\tilde{n})}\|b\|_{L^{\infty}(\mathbf{Z})}^{2 \tilde{n}}\|h\|_{L^{1}\left(\mathbf{Z}^{\tilde{n}}\right)}\left\|h^{\prime}\right\|_{L^{1}\left(\mathbf{Z}^{\tilde{n}}\right)}<\infty$. Finally, if $\tilde{n}=\tilde{m}$, then

$$
\begin{aligned}
I_{(p)_{n}}=\sum_{s_{1}, \ldots, s_{n}} & \left|h^{\prime}\left(s_{1}, \ldots, s_{\tilde{n}}\right) h\left(s_{\tilde{n}+1}, \ldots, s_{n}\right)\right| \\
& \times \sum_{t}\left|b\left(t+s_{1}\right) \cdots b\left(t+s_{\tilde{n}}\right) b\left(s_{\tilde{n}+1}-t\right) \cdots b\left(s_{n}-t\right)\right|,
\end{aligned}
$$

where the last sum does not exceed $\|b\|_{L^{2}(\mathbf{Z})}^{2}\|b\|_{L^{\infty}(\mathbf{Z})}^{2(\tilde{n}-1)}$. This proves (3.9).

It remains to show the representation (3.10). Without loss of generality, let $\gamma=\left(U,(V)_{r}\right)$ be such that $U \cap \bigcup_{p=1}^{m} W_{p}=\{(1,1), \ldots,(\tilde{m}, 1)\}(\tilde{m} \leq m)$. Then

$$
\begin{aligned}
& h^{\gamma}\left(s_{i, j}:(i, j) \in \bigcup_{p=m+1}^{k} W_{p} \cap U\right) \\
& \quad=\sum_{\tilde{s}_{1}, \ldots, \tilde{s}_{r}} \prod_{p=\tilde{m}+1}^{k} q_{p}\left(s_{i, j}:(i, j) \in W_{p}\right) \mathbf{1}\left(s_{i, j}=\tilde{s}_{l}:(i, j) \in V_{l}, l=1, \ldots, r\right) .
\end{aligned}
$$

Hence and from $q_{p} \in L^{1}\left(\mathbf{Z}^{n_{p}}\right), m+1 \leq p \leq k$, the relation $h^{\gamma} \in L^{1}\left(\mathbf{Z}^{\tilde{n}_{\gamma}}\right)$ follows easily. This ends the proof of Lemma 3.2 and of Theorem 1, too.

\section{Proofs of Theorems 2 and 3.}

4.1. Proof of Theorem 2. Without loss of generality, one can assume $b_{i}=$ $0, i=0,1, \ldots, m$. Note for each $t \in \mathbf{Z}, Y_{t}=\sum_{i \leq t-m-1} b_{t-i} \zeta_{i}$ is independent of $Z_{t}=V\left(\zeta_{t}, \ldots, \zeta_{t-m}\right)$. To simplify the notation, we shall also assume $E\left\{Y_{0}^{2}\right\}=$ 1. Observe that the p.d.f. $F(x)=P\left\{X_{0} \leq x\right\}$ is infinitely differentiable, being the convolution of the p.d.f. $F_{Z}(x)=P\left\{Z_{0} \leq x\right\}$ with the standard Gaussian p.d.f. $F_{Y}(x)=P\left\{Y_{0} \leq x\right\}$. As

$$
\begin{aligned}
E\left\{G\left(X_{0}+c\right)\right\} & =\int_{\mathbf{R}} \int_{\mathbf{R}} G(y+z+c) d F_{Y}(y) d F_{Z}(z) \\
& =\frac{1}{\sqrt{2 \pi}} \int_{\mathbf{R}}\left\{\int_{\mathbf{R}} G(y+z) d F_{Z}(z)\right\} e^{-(y-c)^{2} / 2} d y,
\end{aligned}
$$


so the right-hand side of (4.1) is also infinitely differentiable in $c$, under the sign of the integral. Consequently, Appell coefficients $a_{k}$ (1.12) are well defined for any $k \geq 0$ and we obtain

$$
a_{k}=\int_{\mathbf{R}} c_{k}(z) d F_{Z}(z)=E\left\{c_{k}\left(Z_{0}\right)\right\}
$$

where

$$
c_{k}(z)=\int_{\mathbf{R}} G(y+z) H_{k}(y) d F_{Y}(y)
$$

Furthermore,

$$
G\left(Y_{t}+z\right)=\sum_{k=0}^{\infty} \frac{c_{k}(z)}{k !} H_{k}\left(Y_{t}\right)
$$

where the series converges in $L^{2}(\Omega)$, for each $z \in \mathbf{R}$ fixed. We claim that

$$
G\left(X_{t}\right)=G\left(Y_{t}+Z_{t}\right)=\sum_{k=0}^{\infty} \frac{c_{k}\left(Z_{t}\right)}{k !} H_{k}\left(Y_{t}\right) \equiv \sum_{k=0}^{\infty} \frac{c_{k}\left(Z_{t}\right)}{k !}: Y_{t}^{, k}:
$$

where the series converges in $L^{2}(\Omega)$. Indeed,

$$
\begin{aligned}
& E\left(G\left(Y_{t}+Z_{t}\right)-\sum_{k=0}^{K} \frac{c_{k}\left(Z_{t}\right)}{k !} H_{k}\left(Y_{t}\right)\right)^{2} \\
& \quad=\int_{\mathbf{R}} \int_{\mathbf{R}}\left(G(y+z)-\sum_{k=0}^{K} \frac{c_{k}(z)}{k !} H_{k}(y)\right)^{2} d F_{Y}(y) d F_{Z}(z) \\
& =\int_{\mathbf{R}} \psi_{K}(z) d F_{Z}(z),
\end{aligned}
$$

where $\psi_{K}(z)=\sum_{k=K+1}^{\infty} c_{k}^{2}(z) / k$ !. According to (4.4), for each $z \in \mathbf{R}, \psi_{K}(z) \rightarrow 0$ $(K \rightarrow \infty)$, and $\psi_{K}(z) \leq \bar{\psi}(z)=E\left\{G^{2}\left(Y_{t}+z\right)\right\}$, where $\bar{\psi}(z)$ is integrable: $\int_{\mathbf{R}} \bar{\psi}(z) d F_{Z}(z)=E\left\{G^{2}\left(X_{t}\right)\right\}<\infty$. Therefore, $\lim _{K \rightarrow \infty} \int_{\mathbf{R}} \psi_{K}(z) d F_{Z}(z)=0$ according to the Lebesgue dominated convergence theorem, thereby proving the claim.

Let us show that

$$
R_{N} \equiv E\left(\sum_{t=1}^{N} G\left(X_{t}\right)-\frac{1}{k^{*} !} c_{k^{*}}\left(Z_{t}\right): Y_{t}^{, k^{*}}:\right)^{2}=o\left(D_{N, k^{*}}^{2}\right) .
$$

By (4.5),

$$
R_{N}=\sum_{t, t^{\prime}=1}^{N} \sum_{k, k^{\prime} \neq k^{*}} \rho_{t, k, t^{\prime}, k^{\prime}} / k ! k^{\prime} !
$$

where

$$
\rho_{t, k, t^{\prime}, k^{\prime}}=E\left\{c_{k}\left(Z_{t}\right) c_{k^{\prime}}\left(Z_{t^{\prime}}\right): Y_{t}^{, k}:: Y_{t^{\prime}}^{, k^{\prime}}:\right\} .
$$


Let $t<t^{\prime},\left|t^{\prime}-t\right|>m$. Put $\mathscr{F}_{[s, t]}=\sigma\left\{\zeta_{i}: s \leq i \leq t\right\}$. Then

$$
\rho_{t, k, t^{\prime}, k^{\prime}}=E\left\{c_{k}\left(Z_{t}\right) c_{k^{\prime}}\left(Z_{t^{\prime}}\right) E\left\{: Y_{t}^{, k}:: Y_{t^{\prime}}^{, k^{\prime}}: \mid \mathscr{G}_{t, t^{\prime}}\right\}\right\} \text {. }
$$

where $\mathscr{G}_{t, t^{\prime}}=\mathscr{F}_{[t-m, t]} \cup \mathscr{F}_{\left[t^{\prime}-m, t^{\prime}\right]}$. Write $Y_{t^{\prime}}=\tilde{Y}_{1}+\tilde{Y}_{2}$, where

$$
\tilde{Y}_{1}=\sum_{i=t-m}^{t} b_{t^{\prime}-i} \zeta_{i}, \quad \tilde{Y}_{2}=\sum_{i=-\infty}^{t-m-1} b_{t^{\prime}-i} \zeta_{i}+\sum_{i=t+1}^{t^{\prime}-m-1} b_{t^{\prime}-i} \zeta_{i} .
$$

Note that $\tilde{Y}_{1}$ is measurable with respect to $\mathscr{G}_{t, t^{\prime}}$, while $Y_{t}=\sum_{i=-\infty}^{t-m-1} b_{t-i} \zeta_{i}$ and $\tilde{Y}_{2}$ are independent of $\mathscr{I}_{t t^{\prime}}$. Therefore the conditional expectation in (4.8) can be rewritten as

$$
\begin{aligned}
E\left\{: Y_{t}^{, k}:: Y_{t^{\prime}}^{, k^{\prime}}: \mid \mathscr{G}_{t, t^{\prime}}\right\} & =E\left\{: Y_{t}^{, k}::\left(\tilde{Y}_{1}+\tilde{Y}_{2}\right)^{, k^{\prime}}: \mid \mathscr{G}_{t, t^{\prime}}\right\} \\
& =\sum_{l=0}^{k^{\prime}}\left(\begin{array}{c}
k^{\prime} \\
l
\end{array}\right): \tilde{Y}_{1}^{, l}: E\left\{: Y_{t}^{, k}:: \tilde{Y}_{2}^{, k^{\prime}-l}:\right\} .
\end{aligned}
$$

Here,

$$
E\left\{: Y_{t}^{, k}:: \tilde{Y}_{2}^{, k^{\prime}-l}:\right\}= \begin{cases}0, & \text { if } k \neq k^{\prime}-l \\ k ! r_{t^{\prime}-t}^{k}, & \text { if } k=k^{\prime}-l\end{cases}
$$

where

$$
r_{t^{\prime}-t}=E\left\{Y_{t} Y_{t^{\prime}}\right\}=E\left\{Y_{t} \tilde{Y}_{2}\right\}=\sum_{i=m+1}^{\infty} b_{i} b_{t^{\prime}-t+i}, \quad t^{\prime}>t+m .
$$

Consequently,

$$
E\left\{: Y_{t}^{, k}:: Y_{t^{\prime}}^{, k^{\prime}}: \mid \mathscr{G}_{t, t^{\prime}}\right\}=\left(\begin{array}{c}
k^{\prime} \\
k^{\prime}-k
\end{array}\right) k ! r_{t^{\prime}-t}^{k}: \tilde{Y}_{1}^{, k^{\prime}-k}: \mathbf{1}\left(k \leq k^{\prime}\right) .
$$

Substituting the last expression into (4.8) and using (4.2), the independence of $Z_{t^{\prime}}$ from $Z_{t}, \tilde{Y}_{1}$, for $t^{\prime}>t+m$, one obtains

$$
\rho_{t, k, t^{\prime}, k^{\prime}}=\left(\begin{array}{c}
k^{\prime} \\
k^{\prime}-k
\end{array}\right) k ! r_{t^{\prime}-t}^{k} a_{k^{\prime}} E\left\{c_{k}\left(Z_{t}\right): \tilde{Y}_{1}^{, k^{\prime}-k}:\right\} \mathbf{1}\left(k \leq k^{\prime}\right) .
$$

Denote

$$
\left\|c_{k}\right\|^{2}=\int_{\mathbf{R}} c_{k}^{2}(z) d F_{Z}(z)=E\left\{c_{k}^{2}\left(Z_{0}\right)\right\}, \quad \beta_{t^{\prime}-t}^{2}=E\left\{\tilde{Y}_{1}^{2}\right\}=\sum_{i=t-m}^{t} b_{t^{\prime}-i}^{2} .
$$

As $\left|a_{k}\right| \leq\left\|c_{k}\right\|$, from (4.8) by the Cauchy-Schwarz inequality we obtain the bound

$$
\left|\rho_{t, k, t^{\prime}, k^{\prime}}\right| \leq \frac{k^{\prime} !}{\left(\left(k^{\prime}-k\right) !\right)^{1 / 2}}\left|r_{t^{\prime}-t}\right|^{k}\left\|c_{k}\right\|\left\|c_{k^{\prime}}\right\|\left|\beta_{t^{\prime}-t}\right|^{k^{\prime}-k} \mathbf{1}\left(k \leq k^{\prime}\right) .
$$

Let $M>m$ be a fixed integer which will be specified below. Write $\sum_{t, t^{\prime}}^{N, M}$ for the sum over $t, t^{\prime}=1, \ldots, N, t \leq t^{\prime}-M$. Then

$$
\sum_{t, t^{\prime}}^{N, M} \sum_{k, k^{\prime} \neq k^{*}} \frac{\rho_{t, k, t^{\prime}, k^{\prime}}}{k ! k^{\prime} !}=\Sigma_{1}+\Sigma_{2},
$$


where

$$
\begin{aligned}
& \Sigma_{1}=\sum_{t, t^{\prime}}^{N, M} \sum_{k^{*}<k \leq k^{\prime}} \rho_{t, k, t^{\prime}, k^{\prime}} / k ! k^{\prime} !, \\
& \Sigma_{2}=\sum_{t, t^{\prime}}^{N, M} \sum_{0 \leq k<k^{*}<k^{\prime}} \rho_{t, k, t^{\prime}, k^{\prime}} / k ! k^{\prime} !,
\end{aligned}
$$

and where we used the fact that $\rho_{t, k, t^{\prime}, k^{\prime}}=0$ for $k^{\prime}<k^{*}$, which follows from (4.9) and $a_{k^{\prime}}=0, k^{\prime}<k^{*}$. Let us show

$$
\Sigma_{i}=o\left(D_{N, k^{*}}^{2}\right), \quad i=1,2 .
$$

Consider $\Sigma_{1}$. By (4.10),

$$
\left|\Sigma_{1}\right| \leq \sum_{t, t^{\prime}}^{N, M} \sum_{k=k^{*}+1}^{\infty} \frac{\left|r_{t^{\prime}-t}\right|^{k}\left\|c_{k}\right\|}{(k !)^{1 / 2}} \sum_{k^{\prime} \geq k} \frac{\left\|c_{k^{\prime}}\right\|}{\left(k !\left(k^{\prime}-k\right) !\right)^{1 / 2}}\left|\beta_{t^{\prime}-t}\right|^{k^{\prime}-k} .
$$

Note that $k !\left(k^{\prime}-k\right) ! \geq 2^{-k^{\prime}} k^{\prime}$ ! for any $k^{\prime} \geq k \geq 0$. Without loss of generality, one can assume $M$ so large that $\left|2^{1 / 2} \beta_{t^{\prime}-t}\right|<2^{-1 / 2}$ for all $\left|t^{\prime}-t\right|>M$. Then the last sum in (4.12) does not exceed

$$
2^{k / 2} \sum_{k^{\prime} \geq k} \frac{\left\|c_{k^{\prime}}\right\|}{\left(k^{\prime} !\right)^{1 / 2}}\left|2^{1 / 2} \beta_{t^{\prime}-t}\right|^{k^{\prime}-k} \leq 2^{1+k / 2}\|G\|,
$$

where

$$
\|G\|^{2}=E\left\{G^{2}\left(X_{0}\right)\right\}=\sum_{j=0}^{\infty}\left\|c_{j}\right\|^{2} / j !<\infty
$$

Consequently,

$$
\left|\Sigma_{1}\right| \leq 2\|G\| \sum_{t, t^{\prime}}^{N, M} \sum_{k=k^{*}+1}^{\infty} \frac{\left|r_{t^{\prime}-t}\right|^{k}\left\|c_{k}\right\| 2^{k / 2}}{(k !)^{1 / 2}} .
$$

According to (1.1), for any $\theta^{\prime}<\theta$ one can find $M<\infty$ such that $\left|r_{t^{\prime}-t}\right| \leq$ $\left|t^{\prime}-t\right|^{-\theta^{\prime}},\left|t^{\prime}-t\right|>M$. Let $\theta^{\prime \prime}<\theta^{\prime}, \delta=\theta^{\prime}-\theta^{\prime \prime}>0$. Then

$$
\left|r_{t^{\prime}-t}\right|^{k} \leq\left|t^{\prime}-t\right|^{-\left(k^{*}+1\right) \theta^{\prime \prime}}\left|t^{\prime}-t\right|^{-k \delta},
$$

$k \geq k^{*}+1$. Choose now $\theta^{\prime \prime}<\theta^{\prime}$ so that $\left(k^{*}+1\right) \theta^{\prime \prime}>k^{*} \theta$ and further, assume $M$ so large that $2^{1 / 2}\left|t^{\prime}-t\right|^{-\delta}<2^{-1 / 2}$ for $\left|t^{\prime}-t\right|>M$. Then from (4.13) one obtains

$$
\left|\Sigma_{1}\right| \leq 4\|G\|^{2} \sum_{t, t^{\prime}}^{N, M}\left|t^{\prime}-t\right|^{-\left(k^{*}+1\right) \theta^{\prime \prime}}=o\left(D_{N, k^{*}}^{2}\right) .
$$

Next, consider $\Sigma_{2}$. We have

$$
\left|\Sigma_{2}\right| \leq C \sum_{t, t^{\prime}}^{N, M}\left|\beta_{t^{\prime}-t}\right| \sum_{k^{\prime}>k^{*}} \frac{\left\|c_{k^{\prime}}\right\|}{\left(k^{\prime} !\right)^{1 / 2}}\left|\beta_{t^{\prime}-t}\right|^{k^{\prime}-k^{*}},
$$

where $C<\infty$ is a constant. Choose $M$ so that $\left|\beta_{t^{\prime}-t}\right|<2^{-1 / 2},\left|t^{\prime}-t\right|>M$ and use the fact that $\left|\beta_{t^{\prime}-t}\right|=O\left(b_{t^{\prime}-t}^{2}\right)$, where $\sum_{t \geq 0} b_{t}^{2}<\infty$, to conclude that $\left|\Sigma_{2}\right| \leq C\|G\| N=O(N)=o\left(D_{N, k^{*}}^{2}\right)$. 
To complete the proof of (4.5), it suffices to note that for each $M<\infty$ fixed, and with $\tilde{G}_{t}=G\left(X_{t}\right)-\left(k^{*} !\right)^{-1} c_{k^{*}}\left(Z_{t}\right): Y_{t}^{, k^{*}}$ :, one has

$$
\sum_{t, t^{\prime}=1, \ldots, N:\left|t^{\prime}-t\right| \leq M}\left|\operatorname{Cov}\left(\tilde{G}_{t}, \tilde{G}_{t^{\prime}}\right)\right|=O(N)
$$

provided $E\left\{\tilde{G}_{0}^{2}\right\}<\infty$. The last inequality holds by $E\left\{G^{2}\left(X_{0}\right)\right\}<\infty$ and, $Y_{0}$, $Z_{0}$ being independent, by $E\left\{c_{k}^{2}\left(Z_{0}\right)\left(: Y_{0}^{, k}:\right)^{2}\right\}=E\left\{c_{k}^{2}\left(Z_{0}\right)\right\} E\left(: Y_{0}^{, k}:\right)^{2}=$ $k !\left\|c_{k}\right\|^{2}<\infty$.

With (4.5) in mind, Theorem 2 follows from Taqqu (1979) and

$$
E\left(\sum_{t=1}^{N} \bar{c}_{k^{*}}\left(Z_{t}\right) H_{k^{*}}\left(Y_{t}\right)\right)^{2}=O(N),
$$

where $\bar{c}_{k}\left(Z_{t}\right)=c_{k}\left(Z_{t}\right)-E\left\{c_{k}\left(Z_{t}\right)\right\}=c_{k}\left(Z_{t}\right)-a_{k}$. Clearly, (4.14) is a consequence of the orthogonality $\operatorname{Cov}\left(\bar{c}_{k}\left(Z_{t}\right) H_{k}\left(Y_{t}\right), \bar{c}_{k}\left(Z_{t^{\prime}}\right) H_{k}\left(Y_{t^{\prime}}\right)\right)=0,\left|t^{\prime}-t\right|>$ $m$. For $t^{\prime}-m>t$, the last property follows from $E\left\{\bar{c}_{k}\left(Z_{t^{\prime}}\right)\right\}=0$ and the fact that $Z_{t^{\prime}}$ is independent of $Z_{t}, Y_{t}, Y_{t^{\prime}}$. This ends the proof of Theorem 2.

4.2. Proof of Theorem 3. The proof is very similar to and actually simpler than the proof of Theorem 2, so we just give a brief outline.

Let $F_{Y}(x), F_{Z}(x)$ be the same p.d.f. as above. Exactly as in the proof of Theorem 2, one can show that Appell coefficients $a_{k}, k \geq 0$ are well defined and given by (4.2)-(4.3). In a similar way, one can show the relation (4.5). The only place where we need condition (1.18) is to prove (4.14). By the independence of $Y_{t}, t \in \mathbf{Z}$ and $Z_{t}, t \in \mathbf{Z}$, one obtains

$$
\begin{aligned}
\sum_{t \in \mathbf{Z}} \mid E & \left\{\bar{c}_{k}\left(Z_{0}\right) \bar{c}_{k}\left(Z_{t}\right) H_{k}\left(Y_{0}\right) H_{k}\left(Y_{t}\right)\right\} \mid \\
& \leq E\left\{H_{k}^{2}\left(Y_{0}\right)\right\} \sum_{t \in \mathbf{Z}}\left|E\left\{\bar{c}_{k}\left(Z_{0}\right) \bar{c}_{k}\left(Z_{t}\right)\right\}\right|<\infty
\end{aligned}
$$

[see (1.18)], as $E\left\{\bar{c}_{k}^{2}\left(Z_{0}\right)\right\}<\infty$. This proves (4.14) and Theorem 3 .

Below, $\alpha_{N} \sim \beta_{N}$ means $\lim _{N \rightarrow \infty} \alpha_{N} / \beta_{N}=1$. Put $S_{N}^{(G)}=S_{N}^{(G)}(1)$.

COROLlaRY 4.1. Let $G(x), X_{t}=Y_{t}+Z_{t}, Y_{t}, Z_{t}$ satisfy the assumptions of either Theorems 1, 2 or 3. Then

$$
\operatorname{Var}\left(S_{N}^{(G)}\right) \sim a_{k^{*}}^{2} D_{N, k^{*}}^{2}
$$

Proof. Write $G\left(X_{t}\right)=G_{1, t}+G_{2, t}$, where $G_{1, t}=\left(k^{*}\right)^{-1} a_{k^{*}}: Y_{t}^{, k^{*}}:$ It is well known that $\operatorname{Var}\left(\sum_{t=1}^{N} G_{1, t}\right) \sim a_{k^{*}}^{2} D_{N, k^{*}}^{2}$; see, for example, Taqqu (1979) or Surgailis (1982) in the case when $\left\{Y_{t}\right\}$ is Gaussian or linear, respectively. Then the corollary follows from

$$
\operatorname{Var}\left(\sum_{t=1}^{N} G_{2, t}\right)=o\left(D_{N, k^{*}}^{2}\right) .
$$


For $G(x), X_{t}, Y_{t}, Z_{t}$ as in Theorem 1 (respectively, as in Theorem 2 or 3), (4.15) follows from (3.7) and Lemma 3.2 [respectively, from (4.5) and (4.14)].

\section{APPENDIX}

We present below the proofs of Lemmas 2.1-2.3.

ProOF OF LEMMA 2.1. We prove the lemma by induction in the number $|W|$ of elements of $W$. To that end, we need the recursive formula

$$
: Y^{W}:=Y_{j_{1}}: Y^{, W_{1}}:-\sum_{U \subseteq W_{1}}: Y^{, U}: \chi\left(Y^{, W \backslash U}\right),
$$

where $j_{1} \in W$ is arbitrary, and $W_{1}=W \backslash\left\{j_{1}\right\}$. (A.1) can be proved as follows. Note, by definition (2.1),

(A.2) $: Y^{, W}:=Y_{j_{1}}: Y^{, W_{1}}:-(-i)^{|W|} \partial^{\left|W_{1}\right|}\left(f_{1}\left(\mathbf{z}_{1}\right) f_{2}\left(\mathbf{z}_{1}\right)\right) /\left.\partial z^{W_{1}}\right|_{\mathbf{z}_{1}=0}$,

where $\partial^{|U|} / \partial z^{U}=\prod_{j \in U} \partial / \partial z_{j}, \mathbf{z}_{1}=\left(z_{j}: j \in W_{1}\right) \in \mathbf{R}^{\left|W_{1}\right|}$, and where

$$
\begin{aligned}
& f_{1}\left(\mathbf{z}_{1}\right)=\exp \left\{i \sum_{j \in W_{1}} z_{j} Y_{j}\right\} / E \exp \left\{i \sum_{j \in W_{1}} z_{j} Y_{j}\right\}, \\
& f_{2}\left(\mathbf{z}_{1}\right)=\partial \log E \exp \left\{i \sum_{j \in W} z_{j} Y_{j}\right\} /\left.\partial z_{j_{1}}\right|_{z_{j_{1}}=0} .
\end{aligned}
$$

Next, observe, that for any $U \subseteq W_{1}$,

$$
\begin{aligned}
& \partial^{|U|} f_{1}\left(\mathbf{z}_{1}\right) /\left.\partial z^{U}\right|_{\mathbf{z}_{1}=0}=i^{|U|}: Y, U \\
& \partial^{|U|} f_{2}\left(\mathbf{z}_{1}\right) /\left.\partial z^{U}\right|_{\mathbf{z}_{1}=0}=i^{|U|+1} \chi\left(Y^{, U \cup\left\{j_{1}\right\}}\right) .
\end{aligned}
$$

Now, (A.1) follows from (A.2)-(A.4) and the differentiation rule $\partial^{\left|W_{1}\right|}\left(f_{1} f_{2}\right) /$ $\partial z^{W_{1}}=\sum_{U \subseteq W_{1}}\left(\partial^{|U|} f_{1} / \partial z^{U}\right)\left(\partial^{\left|W_{1} \backslash U\right|} f_{2} / \partial z^{W_{1} \backslash U}\right)$.

Let us turn to the proof of (2.6a) and (2.6b). Observe, the lemma holds for $|W|=1$. Assume that (2.6a) is true for any subset $W_{1}=W \backslash\left\{j_{1}\right\} \subset W$. Then, using (A.1),

$$
\begin{aligned}
Y^{W}= & Y_{j_{1}} Y^{W_{1}}=\sum_{U \subseteq W_{1}} Y_{j_{1}}: Y^{, U}: \sum_{\mathscr{V}_{W_{1} \backslash U}} \chi\left(Y^{, V_{1}}\right) \cdots \chi\left(Y^{, V_{r}}\right) \\
= & \sum_{U \subseteq W_{1}}: Y^{, U \cup\left\{j_{1}\right\}}: \sum_{\mathscr{V}_{W_{1} \backslash U}} \chi\left(Y^{, V_{1}}\right) \cdots \chi\left(Y^{, V_{r}}\right) \\
& +\sum_{U \subseteq W_{1}} \sum_{\tilde{U} \subseteq U}: Y^{, \tilde{U}}: \sum_{\mathscr{I}_{W_{1} \backslash U}} \chi\left(Y^{, V_{1}}\right) \cdots \chi\left(Y^{, V_{r}}\right) \chi\left(Y,\left\{j_{1}\right\} \cup U \backslash \tilde{U}\right) .
\end{aligned}
$$

Hence, by changing the order of summation over $U$ and $\tilde{U},(2.6$ a) follows, while (2.6b) follows from the well-known relation between moments and cumulants,

$$
E\left\{Y^{W}\right\}=\sum_{(V)_{r} \in \mathcal{V}_{W}} \chi\left(Y^{, V_{1}}\right) \cdots \chi\left(Y^{, V_{r}}\right) .
$$

This proves Lemma 2.1. 
Proof of LemMa 2.2. We prove the lemma by induction in $|W|$. Applying Lemma 2.1, (2.6a) to the product $Y^{W}=\prod_{i=1}^{k} Y^{W_{i}}$ and to each $Y^{W_{i}}, i=1, \ldots, k$ separately, one obtains

$$
\sum_{U \subseteq W}: Y^{, U}: E\left\{Y^{W \backslash U}\right\}=\sum_{(\tilde{U})_{k}} \prod_{i=1}^{k}: Y^{, \tilde{U}_{i}}: E\left\{Y^{W_{i} \backslash \tilde{U}_{i}}\right\}+\prod_{i=1}^{k}: Y^{, W_{i}}
$$

where the sum $\sum_{(\tilde{U})_{k}}$ is taken over all $\tilde{U}_{1} \subseteq W_{1}, \ldots, \tilde{U}_{k} \subseteq W_{k}$ such that $\tilde{U} \equiv \bigcup_{i=1}^{k} \tilde{U}_{i} \neq W$. As $|\tilde{U}|<|W|$, by the inductive assumption one obtains

$$
\begin{aligned}
\sum_{U \subseteq W}: Y^{, U}: E\left\{Y^{W \backslash U}\right\} & \\
= & \sum_{(\tilde{U})_{k}} \sum_{U \subseteq \tilde{U}}: Y^{, U}: \sum_{(V)_{r} \in \mathscr{Y}_{\tilde{U} \backslash U}^{*}} \chi\left(Y^{,} V_{1}\right) \cdots \chi\left(Y^{,} V_{r}\right) \prod_{i=1}^{k} E\left\{Y^{W_{1} \backslash \tilde{U}_{i}}\right\} \\
& +\prod_{i=1}^{k}: Y^{, W_{i}}:
\end{aligned}
$$

where, for $U^{\prime} \subseteq W, \mathscr{V}_{U^{\prime}}^{*}$ is the set of all partitions $(V)_{r} \in \mathscr{V}_{U^{\prime}}$ without flat edges. We claim that for each $U \subseteq W$,

$$
\begin{aligned}
\alpha_{U} & \equiv E\left\{Y^{W \backslash U}\right\}-\sum_{\tilde{U}: U \subseteq \tilde{U} \subset W} \sum_{(V)_{r} \in \mathscr{V}_{\tilde{U} \backslash U}^{*}} \chi\left(Y^{, V_{1}}\right) \cdots \chi\left(Y^{, V_{r}}\right) \prod_{i=1}^{k} E\left\{Y^{W_{i} \backslash \tilde{U}}\right\} \\
& =\sum_{(V)_{r} \in \mathscr{Y}_{W \backslash U}^{*}} \chi\left(Y^{, V_{1}}\right) \cdots \chi\left(Y^{,} V_{r}\right) .
\end{aligned}
$$

This proves the induction step, as $\prod_{i=1}^{k}: Y^{,} W_{i}:=\sum_{U \subseteq W} \alpha_{U}: Y, U$ : according to (A.6). It remains to show the claim (A.7). To do this, split the sum $\sum_{(V)_{r} \in \mathcal{V}_{W \backslash U}} \prod_{s=1}^{r} \chi\left(Y^{,}, V_{s}\right)=E\left\{Y^{W \backslash U}\right\}$ into two parts $\sum_{(V)_{r} \in \mathcal{V}_{W \backslash U}^{*}} \prod_{s=1}^{r} \chi\left(Y^{, V_{s}}\right)+$ $\sum_{(V)_{r} \varkappa_{W W U}^{*}} \prod_{s=1}^{r} \chi\left(Y, V_{s}\right) \equiv \sum_{1}+\sum_{2}$. Then, rewrite

$$
\sum_{2}=\sum_{\tilde{U}: U \subseteq \tilde{U} \subset W} \sum_{(V)_{r} \in \mathcal{V}_{U}^{*} \backslash U} \prod_{s=1}^{r} \chi\left(Y^{,} V_{s}\right) \prod_{i=1}^{k} \sum_{\left(V^{\prime}\right)_{r^{\prime}} \in \mathscr{V}_{W_{i} \backslash \tilde{U}}} \prod_{s^{\prime}=1}^{r^{\prime}} \chi\left(Y^{, V_{s^{\prime}}^{\prime}}\right) .
$$

Here, the last sum on the right-hand side equals $E\left\{Y^{W_{i} \backslash \tilde{U}}\right\}$, for each $i=$ $1, \ldots, k$, according to (A.5).

This proves (2.7a). (2.8) follows by taking expectations of both sides of (2.7a) and using (2.2). Finally, (2.7b) follows from (2.7a) and (2.8). Lemma 2.2 is proved.

Proof OF LemMa 2.3. Note, by repeated use of the Cauchy-Schwarz inequality, that $q^{\gamma} \in L^{2}\left(\mathbf{Z}^{n_{\gamma}}\right)$. Indeed, it suffices to check this property in the case when $\left|V_{i} \cap W_{j}\right|=1$ for every $i=1, \ldots, r, j=1, \ldots, k$. Assume first $r=1$, that is, that $\gamma=\left(U, V_{1}\right)$ has a single connected edge, say, $V_{1}=\left\{(i, 1): 1 \leq i \leq k^{\prime}\right\}$, where $2 \leq k^{\prime} \leq k$. Then $\left|q^{\gamma}\right| \leq\left|\chi_{k^{\prime}}\right| f_{1} f_{2} f_{3}$, where 
$f_{1}=\left(\sum_{\tilde{s}} q_{1}^{2}\left(\tilde{s}, s_{1,2}, \ldots, s_{1, n_{1}}\right)\right)^{1 / 2}, \quad f_{2}=\left(\sum_{\tilde{s}} \prod_{i=2}^{k^{\prime}} q_{i}^{2}\left(\tilde{s}, s_{i, 1}, \ldots, s_{i, n_{i}}\right)\right)^{1 / 2} \quad$ and $f_{3}=\prod_{i=k^{\prime}+1}^{k}\left|q_{i}\left(s_{i, 1}, \ldots, s_{i, n_{i}}\right)\right|$, whence this property clearly follows. In the case $r \geq 2$, the above inequality can be used to reduce the number of connected edges by 1 , and the property follows by induction in $r$.

It suffices to show (2.16) and (2.17) for $q_{i}\left(s_{1}, \ldots, s_{n_{i}}\right)$ vanishing outside a finite set of integer points, $i=1, \ldots, k$. In this case, $\mathscr{A}_{n_{i}}\left(q_{i}\right), i=1, \ldots, k$ are finite sums, and

$$
\prod_{i=1}^{k} \mathscr{A}_{n_{i}}\left(q_{i}\right)=\sum_{s_{i, j} \in \mathbf{Z}:(i, j) \in W} \tilde{q}\left(s_{i, j}:(i, j) \in W\right) \prod_{i=1}^{k}: \zeta, W_{i}:,
$$

where $\tilde{q}$ is given by (2.13). By Lemma 2.2 ,

$$
\prod_{i=1}^{k}: \zeta, W_{i}:=\sum_{\gamma=\left(U,(V)_{p}\right) \in \Gamma_{(n)_{k}}}: \zeta, U: \prod_{l=1}^{r} \chi\left(\zeta, V_{l}\right) .
$$

Hence (2.16) follows, because $\chi\left(\zeta^{,}, V\right)=0$ unless all $s_{i, j},(i, j) \in V \subseteq W$ are equal. Equation (2.17) follows from (2.16) and $E\left\{\mathscr{\mathscr { A }}_{n}(q)\right\}=0, n \geq 1$. Lemma 2.3 is proved.

Acknowledgments. I thank Paul Doukhan and Liudas Giraitis for discussions and the referee for useful comments. The hospitality and support of IMPA and CNPq is acknowledged.

\section{REFERENCES}

Avram, F. and TAQQU, M. S. (1987). Generalized powers of strongly dependent random variables. Ann. Probab. 15 767-775.

BERAN, J. (1992). Statistical methods for data with long-range dependence (with discussions). Statist. Sci. 7 404-427.

Breuer, P. and MAJOR, P. (1983). Central limit theorem for non-linear functionals of Gaussian fields. J. Multivariate Anal. 13 425-441.

Dobrushin, R. L. (1979). Gaussian and their subordinated self-similar random generalized fields. Ann. Probab. 7 1-28.

Dobrushin, R. L. and MAJOR, P. (1979). Non-central limit theorems for non-linear functionals of Gaussian fields. Z. Wahrsch. Verw. Gebiete 50 27-52.

GIRAitis, L. and SuRGaiLIS, D. (1985). CLT and other limit theorems for functionals of Gaussian processes. Z. Wahrsch. Verw. Gebiete 70 191-212.

GIRAITIS, L. and Surgailis, D. (1986). Multivariate Appell polynomials and the central limit theorem. In Dependence in Probability and Statistics (E. Eberlein and M. S. Taqqu, eds.) 21-71. Birkhäuser, Boston.

GIRAITIS, L. and Surgailis, D. (1989). A limit theorem for polynomials of linear process with long range dependence. Lithuanian Math. J. 29 290-311.

GIRAITIS, L. and TAQQU, M. S. (1997). Limit theorems for bivariate Appell polynomials I: Central limit theorems. Probab. Theory Related Fields 107 359-381.

GIRAITIS, L., TAQQU, M. S. and TERRIN, N. (1998). Limit theorems for bivariate Appell polynomials II: Non-central limit theorems. Probab. Theory Related Fields 110 333-368.

Ho, H. C. and Hsing, T. (1996). On the asymptotic expansion of the empirical process of long memory moving average. Ann. Statist. 24 992-1024. 
Koul, H. and Surgailis, D. (1997). Asymptotic expansion of $M$-estimators with long memory errors. Ann. Statist. 25 818-850.

MAJOR, P. (1981). Multiple Wiener-Itô Integrals. Lecture Notes in Math. 849. Springer, New York. Rosenblatt, M. (1961). Independence and dependence. In Proc. Fourth Berkeley Symp. Math. Statist. Probab. 411-443. Univ. California Press, Berkeley.

SuRGailis, D. (1982). Zones of attraction of self-similar multiple integrals. Lithuanian Math. J. 22 327-340.

SuRGaILIS, D. (1983). On Poisson multiple stochastic integrals and associated equilibrium Markov processes. In Theory and Applications of Random Fields. Lecture Notes in Control and Inform. Sci. 49 233-248. Springer, New York.

TAQQU, M. S. (1978). A representation for self-similar processes. Stochastic Process. Appl. 7 55-64. TAQQU, M. S. (1979). Convergence of integrated processes of arbitrary Hermite rank. Z. Wahrsch. Verw. Gebiete 50 53-83.

VilniUs Institute of Mathematics AND INFORMATICS

AKADEMIJOS 4

2600 VILNIUS

LITHUANIA

E-MAIL: sdonatas@ktl.mii.lt 